\title{
Static quark potential according to the dual-superconductor picture of $\mathrm{QCD}$
}

\author{
M. Baker \\ Department of Physics, University of Washington, Seattle, Washington 98195 \\ J. S. Ball \\ Department of Physics, University of Utah, Salt Lake City, Utah 84112 \\ F. Zachariasen \\ California Institute of Technology, Pasadena, California 91125
}

(Received 16 June 1986)

\begin{abstract}
We use the effective action describing long-range $\mathrm{QCD}$, which predicts that $\mathrm{QCD}$ behaves as a dual superconductor, to derive the interaction energy between two heavy quarks as a function of separation. The dual-superconductor field equations are solved in an approximation in which the boundary between the superconducting vacuum and the region of normal vacuum surrounding the quarks is sharp. Further, non-Abelian effects are neglected. The resulting heavy-quark potential is linear in separation at large separation, and Coulomb-like at small separation. Overall it agrees very well with phenomenologically determined potentials.
\end{abstract}

\section{INTRODUCTION}

The dual-superconductor description of long-range QCD is necessarily expressed in terms of the dual vector potential $C_{\mu}$ rather than the conventional vector potential $A_{\mu}$, as a result of the singularities in $A_{\mu}$. In this language the introduction of color-electric sources is awkward, because the use of dual potentials makes Gauss's law and Ampere's law into kinematic identities without sources. The problem is superficially similar to that encountered in the attempt to describe magnetic monopoles with the ordinary vector potential in electromagnetism. There, monopoles can be introduced at the price of attaching Dirac strings to them, and the strings can be shown to have no observable physical effect if Dirac's quantization condition $e g=2 \pi$ is obeyed. ${ }^{2}$

Electric point charges, however, have no strings. The field of an electric point charge is just a Coulomb field; it is not a dual Dirac monopole with a string. It is not true that $\boldsymbol{\nabla} \cdot \mathbf{E}=0$. Therefore, one must add to the relation between the electric field and the potential a string electric field, which is arranged to precisely cancel the string coming from the dual potential. ${ }^{3}$ This string field has a divergence, and its presence therefore allows Gauss's law, with sources, to be satisfied even when the dual potential is used.

Our purpose here is to introduce heavy-quark sources into the dual-superconductor effective action describing long-range QCD, and to calculate the static quark potential as a function of quark separation. The vehicle for doing this will be the "string fields" mentioned above. The details of how they are used are given in Sec. II.

We make use of a simplifying approximation in carrying out the calculation, in which the regions of superconducting vacuum and normal vacuum are sharply separated. This approximation is described in Secs. III and IV. It leads to a set of equations with features similar to, but not identical with, the conventional MIT bag model. ${ }^{4}$ Consequently one can view this as a derivation of (a modified) bag model from the dual superconductor. The application of the approximation to the quark potential is made in Secs. V, VI, and VII at the Abelian level. The approximate field equations are solved analytically in the limit of large quark separation, where the region of normal vacuum becomes a cylinder, and numerically via a variational calculation for arbitrary separation. For small separations, the potential becomes Coulomb-like, so that the dual superconductor effective action interpolates smoothly all the way to the short-range regime. It may therefore be used as an approximate effective action for QCD at all ranges. We recall that the magnetic permeability giving rise to the effective action is, ${ }^{1}$ in momentum space, $\mu=M^{2} / q^{2}+1 / f^{2}$. At short range, therefore, i.e., as $q^{2} \rightarrow \infty$, the action (1.1) of I becomes simply

$$
L \rightarrow \frac{1}{4 f^{2}} G_{\mu \nu} G_{\mu \nu} .
$$

In this limit we may identify $G_{\mu \nu}$ with $\frac{1}{2} \epsilon_{\mu \nu \lambda \sigma} F_{\lambda \sigma}$ in terms of the ordinary field tensor $F_{\lambda \sigma}$, so that $L$ coincides with the usual QCD Lagrangian provided that $f=1$. Smooth interpolation to the correct short-range limit thus requires that the mass parameters $M$ and $M_{f}$ of I are related by $M_{f}=M$.

In principle, classical colored sources make no sense, since by a gauge transformation one can change sources with, for example, parallel color to sources with antiparallel color. It is in general necessary to quantize the effective action to deal properly with sources. At the Abelian level, however, quantization is not required.

In the Abelian, or small- $g$, limit, the quark sources must be in a color-singlet configuration to have a finite energy. To higher orders in $g$, other configurations are possible since explicit quantum gluons can combine with the quarks so that the entire system forms a singlet. That 
is, the gluons can screen the color of the quarks. Evidently, incorporating these effects requires quantizing the gluon field.

Dual-i.e., color-magnetic-gluons do not, however, individually screen color-electric-i.e., quark-sources. Therefore to properly incorporate the physics of screening in the dual language requires going to all orders in $g$; one or two additional orders of $g$ are not enough. This is not surprising; the electric and magnetic gauge groups are not identical. A single electric gluon together with two quarks in a color octet can make up an overall color singlet, so that screening can occur in order $e^{2}$. But $e \sim 1 / g$; thus all orders in $g$ are involved. We shall not address higher orders in $g$ further in this paper.

The dual superconductor effective action is ${ }^{1}$

$$
\begin{array}{r}
S=\int d^{3} \mathbf{x}\left[N \operatorname { T r } \left[\frac{1}{2} G_{\mu \nu} \widetilde{F}_{\mu \nu}+\frac{1}{4} \widetilde{F}_{\mu \nu} \frac{\mathscr{D}^{2}}{M^{2}} \widetilde{F}_{\mu \nu}\right.\right. \\
\left.\left.-\frac{1}{4 f^{2}} G_{\mu \nu} G_{\mu \nu}\right]-W(\widetilde{F})\right] .
\end{array}
$$

Here

$$
G_{\mu \nu}=\partial_{\mu} C_{v}-\partial_{\nu} C_{\mu}-i g\left[C_{\mu}, C_{v}\right]
$$

and

$$
\mathscr{D}_{\mu}=\partial_{\mu}-i g\left[C_{\mu},\right],
$$

where $C_{\mu}$ is the dual potential. The color notation is $C_{\mu} \equiv C_{\mu}^{a} T^{a}$, and the generators $T^{a}$ are normalized to $\left[T^{a}, T^{b}\right]=i f^{a b c} T^{c}$. Thus $\operatorname{Tr} T^{a} T^{a}=1 / N$ for $\operatorname{SU}(N)$. The field $\widetilde{f}_{\mu v}$ is an auxiliary field, introduced to eliminate operators like $1 / \mathscr{D}^{2}$. We refer to the components of $\widetilde{F}$ as electric and magnetic fields: $E_{i}=-\frac{1}{2} \epsilon_{i j k} \widetilde{F}_{j k}$ and $B_{i}=-\widetilde{F}_{0 i}$, even though these are the true color $\mathbf{E}$ and $\mathbf{B}$ fields only in the Abelian and long-range limit. (Other definitions of the electric and magnetic fields could have been made; for example, we might have called $\mathbf{D}+\mathbf{E}$ the electric field, since the electric energy density [see Eq. (3.12)], at the Abelian level, is given by $\frac{1}{2} \mathbf{D} \cdot(\mathbf{D}+\mathbf{E})$. We have found the present choice to be the most useful intuitively.)

The potential $W(\widetilde{F})$ is a phenomenological term independent of $C_{\mu}$ and containing no derivatives. Spontaneous symmetry breaking is associated with a minimum of $W$ at a nonzero value of $\widetilde{F}$; thus $W$ plays the role of the Higgs potential and $\widetilde{F}$ the role of the Higgs field.

In the past we have analyzed (1.2) only for pure glue with no sources. Among other things we have found quantized tubes of color-electric flux as excitations of the vacuum. ${ }^{1}$ Our purpose here is to relate these flux tubes to heavy-quark sources, to see how they are modified when the sources are close to each other, and to extract the interaction energy between the sources, that is, the static quark potential.

\section{INCORPORATING SOURCES INTO DUAL POTENTIALS}

In sourceless electricity use of the dual potential makes Gauss's law $\boldsymbol{\nabla} \cdot \mathbf{D}=0$ and Ampere's law $\boldsymbol{\nabla} \times \mathbf{H}-\partial_{0} \mathbf{D}=\mathbf{0}$ into kinematic relations automatically satisfied in virtue of the definition of the fields in terms of the potential: $\mathbf{D}=-\boldsymbol{\nabla} \times \mathbf{C}$ and $\mathbf{H}=-\partial_{0} \mathbf{C}-\boldsymbol{\nabla} C_{0}$.

When electric sources are present, these expressions for the fields must be modified. ${ }^{3}$ To introduce an electric charge density $\rho$, for example, one must write

$$
\mathbf{D}=-\boldsymbol{\nabla} \times \mathbf{C}+\mathbf{D}_{s}
$$

and choose the "string field" $\mathbf{D}_{s}$ to satisfy

$$
\boldsymbol{\nabla} \cdot \mathbf{D}_{s}=\rho
$$

so that the electric displacement will obey Gauss's law with sources. For a single point charge $q$ located at the origin, the decomposition (2.1) simply reflects the identity (in spherical coordinates)

$$
\frac{q}{4 \pi r^{2}} \widehat{\mathbf{e}}_{r}=-\nabla \times\left(\frac{q}{4 \pi} \frac{1+\cos \theta}{r \sin \theta} \widehat{\mathbf{e}}_{\phi}\right)+q \delta(x) \delta(y) \theta(z) \widehat{\mathbf{e}}_{z} .
$$

The "string field" $D_{s}$ is

$$
\mathbf{D}_{s}=q \delta(x) \delta(y) \theta(z) \widehat{\mathbf{e}}_{z} ;
$$

it exists only along the positive $z$ axis and serves to cancel the string in $-\boldsymbol{\nabla} \times \mathbf{C}$ which results from the attempt to represent a point charge as the curl of something. Both $-\nabla \times \mathbf{C}$ and $\mathbf{D}_{s}$ contain strings; $\mathbf{D}$ does not. Evidently

$$
\boldsymbol{\nabla} \cdot \mathbf{D}=q \delta^{3}(\mathbf{r})
$$

as required. For two equal and opposite charges located on the $z$ axis at $z= \pm R / 2$, we have

$$
\mathbf{C}=\frac{q}{4 \pi}\left(\frac{1+\cos \theta_{1}}{r_{1} \sin \theta_{1}}-\frac{1+\cos \theta_{2}}{r_{2} \sin \theta_{2}}\right) \widehat{\mathbf{e}}_{\phi},
$$

where $r_{1,2}=\left|\mathbf{r} \mp \frac{1}{2} R \widehat{\mathbf{e}}_{z}\right|$; now

$$
\mathbf{D}_{s}=q \delta(x) \delta(y)[\theta(z-R / 2)-\theta(z+R / 2)] \widehat{\mathbf{e}}_{z}
$$

and the string only joins the two charges and no longer extends to infinity.

The electrostatic action, with sources, becomes

$$
S=\frac{1}{2} \int d^{3} \mathbf{x}\left[-\nabla \times \mathbf{C}(\mathbf{x})+\mathbf{D}_{s}(\mathbf{x})\right]^{2} ;
$$

Maxwell's equations are obtained from this with $\mathbf{C}$ as the independent variable. The extension of all this to include electric current sources is straightforward. ${ }^{3}$

Exactly the same procedure can be followed to introduce sources into the effective action of QCD, expressed in terms of the dual potential. We now write

$$
\mathbf{D}=-\boldsymbol{\nabla} \times \mathbf{C}-\frac{i g}{2}[\mathbf{C}, \times \mathbf{C}]+\mathbf{D}_{s}
$$

with the string field $\mathbf{D}_{s}$ chosen to satisfy

$$
\boldsymbol{\nabla} \cdot \mathbf{D}_{s}+i g\left[\mathbf{C}, \mathbf{D}_{s}\right]=\rho
$$

for a static charge distribution $\rho$. The action has the same form as in the sourceless case: ${ }^{1}$ 


$$
\begin{aligned}
S=\int d^{3} \mathbf{x}\left[N \operatorname { T r } \left[-\mathbf{D} \cdot \mathbf{E}-\frac{1}{2 f^{2}} D^{2}+\frac{1}{2} \mathbf{E} \cdot \frac{\mathscr{D}^{2}}{M^{2}} \mathbf{E}\right.\right. \\
\left.\quad+\mathbf{B} \cdot \mathbf{H}+\frac{1}{2 f^{2}} H^{2}-\frac{1}{2} \mathbf{B} \cdot \frac{\mathscr{D}^{2}}{M^{2}} \mathbf{B}\right] \\
-W(\mathbf{E}, \mathbf{B})],
\end{aligned}
$$

but now (2.8) is to be used to express $D$ in terms of the independent variable $\mathbf{C}$. Again the modifications necessary to add color-electric currents, as well as charges, as sources are straightforward.

\section{THE APPROXIMATE DUAL SUPERCONDUCTOR}

The field equations obtained from (2.10) are a complicated set of coupled nonlinear differential equations. For the source-free case we have studied them earlier in both cylindrical $^{1}$ and spherical ${ }^{5}$ geometries. The basic features of the solutions can be summarized as follows, as can be seen from the graphs of the numerical solutions shown in I. First, far from an excitation a nonperturbative vacuum obtains, characterized by a minimum of the potential $W(\mathbf{E}, \mathbf{B})$ at a nonzero value of the magnetic field $\mathbf{B}=\mathbf{B}_{0}$ and a zero electric field $\mathbf{E}$. The asymptotic vector potential $\mathbf{C}$ vanishes only slowly, like (distance) ${ }^{-1}$, while the scalar potential $C_{0}$ is zero. The vacuum energy density $\epsilon_{\mathrm{vac}}=W\left(0, \mathrm{~B}_{0}\right)$ is negative.

As one approaches the excitation the fields differ from their asymptotic values, but only by amounts vanishing exponentially with distance. This exponential damping is caused by the nonzero value of $W$ which effectively prevents the fields from penetrating the nonperturbative vacuum. Inside the excitation the magnetic field $\mathbf{B}$ becomes small, vanishing at the center. Therefore $W$ vanishes too. All fields are continuous, smooth, functions throughout. The surface of the excitation is a fairly broad region, of dimension comparable to the size of the excitation itself.

These properties suggest that the exact field equations can be replaced by an approximation in which the surface region of the excitation is replaced by a sharp boundary. Outside of the boundary, the energy density is simply $\epsilon_{\mathrm{vac}}$ and the fields have their asymptotic values. Inside, the exact field equations hold except that the potential $W$ is set equal to zero. At the surface the independent fields $C_{t}, C_{0}, E_{t}$, and $B_{n}$ are to be continuous with their asymptotic values, while the normal component of $\mathbf{D}$ and the tangential component of $\mathbf{H}$ vanish.

How much violence such an approximation does to the actual solutions remains to be seen. The true surface of the excitation is, as we have mentioned, not at all sharp. Nevertheless, it appears that all qualitative aspects of the solutions are reasonably well maintained.

It is useful to keep in mind the differences between this approximation and the conventional MIT bag model. ${ }^{5}$ The dual superconductor has a region inside of which the "potential" $W$ (analogous to the Higgs potential in the Abelian Higgs model, ${ }^{6}$ or in the Landau-Ginzburg equations of a relativistic superconductor ${ }^{7}$ ) vanishes, and outside of which $W=\epsilon_{\mathrm{vac}}$ is a constant. In the MIT bag, the bag is a region where the dielectric constant $\epsilon=1$, while outside $\epsilon=0$. Here, in contrast, the dielectric constant (or rather the permeability $\mu=\epsilon^{-1}$ ) is everywhere given by $\mu=M^{2} / \mathscr{D}^{2}+1 / f^{2}$, where $\mathscr{D}_{\mu}$ is the (dual) covariant derivative $\mathscr{D}_{\mu}=\partial_{\mu}-i g\left[C_{\mu},\right]$. (In this respect, the present picture is more like that advocated by Friedberg and Lee, ${ }^{8}$ though that is expressed in terms of the usual vector potential, not the dual one.) Nevertheless, as we will see, many of the properties we find here are similar to those of the MIT bag model.

It is also important to emphasize that the present picture is simply an approximation to the exact field equations following from the effective action (2.10), supposed to describe long-range QCD with sources present. It is not more ad hoc than (2.10) is; if (2.10) indeed can be shown to follow from quantum QCD, then the model described here is a fortiori derivable as a reasonably good approximation to quantum QCD.

To summarize, our approximate field equations are [as obtained from (2.10) by varying with respect to the independent canonical fields $\mathbf{C}, C_{0}, \mathbf{E}$, and $\mathbf{B}$ ].

(i) "Gauss's law"

$$
\frac{1}{f^{2}} \mathscr{D} \cdot \mathbf{H}+\mathscr{D} \cdot \mathbf{B}-i \frac{g}{M^{2}}\left[\mathscr{D}^{0} \mathbf{E}, \cdot \mathbf{E}\right]+i \frac{g}{M^{2}}\left[\mathscr{D}{ }^{0} \mathbf{B}, \cdot \mathbf{B}\right]=0
$$

(ii) "Ampere’s law"

$$
\begin{aligned}
\frac{1}{f^{2}} \mathscr{D} \times \mathbf{D}+\mathscr{D} \times \mathbf{E}+\mathscr{D}{ }_{0} \mathbf{H} & +\mathscr{D}_{0} \mathbf{B}-i \frac{g}{M^{2}}\left[\mathscr{D} E_{i}, E_{i}\right] \\
& +i \frac{g}{M^{2}}\left[\mathscr{D} B_{i}, B_{i}\right]=0 .
\end{aligned}
$$

(iii) The constitutive equations of the medium

$$
\mathbf{H}=\frac{\left(\mathscr{D}_{0}{ }^{2}-\mathscr{D}^{2}\right)}{M^{2}} \mathbf{B}
$$

and

$$
\mathbf{D}=\frac{\left(\mathscr{D}_{0}^{2}-\mathscr{D}^{2}\right)}{M^{2}} \mathbf{E} .
$$

Sources are introduced through the relations between $\mathbf{D}, \mathbf{H}$, and the potentials

$$
\begin{aligned}
& \mathbf{D}=-\nabla \times \mathbf{C}-i \frac{g}{2}[\mathbf{C}, \times \mathbf{C}]+\mathbf{D}_{s}, \\
& \mathbf{H}=-\mathscr{D} C_{0}-\partial_{0} \mathbf{C}+\mathbf{H}_{s},
\end{aligned}
$$

with the "string fields" chosen to satisfy

$$
\begin{aligned}
& \mathscr{D} \cdot \mathbf{D}_{s}=\rho, \\
& \mathscr{D} \times \mathbf{H}_{s}-\mathscr{D}{ }_{0} \mathbf{D}_{s}=\mathbf{j},
\end{aligned}
$$

for any given color-electric charge and current densities $\rho$ and $\mathbf{j}$ which we wish to introduce. In all of the above equations,

$$
\mathscr{D}=\boldsymbol{\nabla}+i g[\mathbf{C},]
$$

and

$$
\mathscr{D}_{0}=\partial_{0}-i g\left[C_{0},\right]
$$


Equations (3.1)-(3.8) hold inside the volume $V$ of perturbative vacuum. Outside, the energy density is $\epsilon_{\mathrm{vac}}<0$. Hence the action (relative to the nonperturbative vacuum) is

$$
\begin{array}{rl}
S=\int_{V} d^{3} \mathbf{x} & N \operatorname{Tr}\left[-\mathbf{D} \cdot \mathbf{E}-\frac{1}{2 f^{2}} \mathbf{D}^{2}+\frac{1}{2} \mathbf{E} \cdot \frac{\mathscr{D}^{2}}{M^{2}} \mathbf{E}+\mathbf{B} \cdot \mathbf{H}\right. \\
& \left.\left.+\frac{1}{2 f^{2}} \mathbf{H}^{2}-\frac{1}{2} \mathbf{B} \cdot \frac{\mathscr{D}^{2}}{M^{2}} \mathbf{B}\right]\right]+\epsilon_{\mathrm{vac}} V
\end{array}
$$

At the surface we have (see the next section)

$$
D_{n}=0, H_{t}=0, E_{t}=0, B_{n}=b,
$$

and the position of the surface is determined by balancing the interior pressure [inside the brackets in (3.11)] against the vacuum pressure $-\epsilon_{\mathrm{vac}}>0$.

As we mentioned in the Introduction, classical colored sources really make no sense except at the Abelian level, due to the fact that they are gauge dependent. For this reason, as well as for simplicity, we will in what follows limit ourselves to the Abelian, or small $g$, limit of Eqs. (3.1)-(3.4). In this limit, then, and for a static problem, the action becomes

$$
\begin{aligned}
S=\int_{V} d^{3} \mathbf{x} N \operatorname{Tr}( & -\mathbf{D} \cdot \mathbf{E}-\frac{1}{2 f^{2}} \mathbf{D}^{2}-\frac{1}{2} \mathbf{E} \cdot \frac{\nabla^{2}}{M^{2}} \mathbf{E} \\
& \left.+\mathbf{B} \cdot \mathbf{H}+\frac{1}{2 f^{2}} \mathbf{H}^{2}+\frac{1}{2} \mathbf{B} \cdot \frac{\nabla^{2}}{M^{2}} \mathbf{B}\right)+\epsilon_{\mathrm{vac}} V
\end{aligned}
$$

[in the exact field equations, the forms $\left(\partial_{i} \mathbf{E}\right)^{2}$ and $-\mathbf{E} \cdot \nabla^{2} \mathbf{E}$ in the energy density are equivalent, since the electric field vanishes at infinity. In the approximate equations, the surface term by which they differ, namely, $\oint E_{i} \nabla E_{i} \cdot d \mathbf{S}$, does not vanish on the surface of the region of normal vacuum. The form given in Eq. (3.12) is the correct one for the approximate equations] and the field equations are

$$
\begin{aligned}
& \frac{1}{f^{2}} \boldsymbol{\nabla} \cdot \mathbf{H}+\boldsymbol{\nabla} \cdot \mathbf{B}=0, \\
& \frac{1}{f^{2}} \boldsymbol{\nabla} \times \mathbf{D}+\boldsymbol{\nabla} \times \mathbf{E}=0, \\
& \mathbf{H}=-\frac{\nabla^{2}}{M^{2}} \mathbf{B}, \\
& \mathbf{D}=-\frac{\nabla^{2}}{M^{2}} \mathbf{E},
\end{aligned}
$$

together with

$$
\begin{aligned}
& \mathbf{D}=-\boldsymbol{\nabla} \times \mathbf{C}+\mathbf{D}_{s}, \\
& \mathbf{H}=-\boldsymbol{\nabla} C_{0}+\mathbf{H}_{s},
\end{aligned}
$$

where

$$
\begin{aligned}
& \boldsymbol{\nabla} \cdot \mathbf{D}_{s}=\rho, \\
& \boldsymbol{\nabla} \times \mathbf{H}_{s}=\mathbf{j} .
\end{aligned}
$$

\section{THE ABELIAN DUAL SUPERCONDUCTOR WITHOUT SOURCES}

As a test of the approximate dual superconductor field equations described in Sec. III, we begin with a discussion of an infinite cylinder with no sources but containing one unit of color-electric flux. For $\mathrm{SU}(2)$ of color the exact solution to this problem is given by $I$. The approximate equations are (3.13)-(3.16). To lowest order in $g$ the electric and magnetic problems decouple.

The electric equations (using the scaled notation of I) are

$$
\begin{aligned}
& \frac{\partial}{\partial \rho} \frac{1}{\rho} \frac{\partial}{\partial \rho} \rho C_{\phi}+\frac{\partial^{2}}{\partial z^{2}} C_{\phi}+\frac{\partial E_{\rho}}{\partial z}-\frac{\partial E_{z}}{\partial \rho}=0, \\
& \frac{\partial}{\partial \rho} \frac{1}{\rho} \frac{\partial}{\partial \rho} \rho E_{\rho}+\frac{\partial^{2}}{\partial z^{2}} E_{\rho}=-\frac{\partial C_{\phi}}{\partial z},
\end{aligned}
$$

and

$$
\frac{1}{\rho} \frac{\partial}{\partial \rho} \rho \frac{\partial}{\partial \rho} E_{z}+\frac{\partial^{2}}{\partial z^{2}} E_{z}=\frac{1}{\rho} \frac{\partial}{\partial \rho} \rho C_{\phi},
$$

and hold for $0 \leq \rho \leq a$, where $a$ is the radius of the cylinder. The relevant solutions to these are

$$
\begin{aligned}
& C_{\phi}=-\frac{\rho}{2 \pi a^{2}}, \\
& E_{\rho}=-\frac{\rho z}{2 \pi a^{2}}
\end{aligned}
$$

[there are additional solutions to Eqs. (4.1) and (4.2), in which $C_{\phi}, E_{\rho} \sim I_{1}(\rho)$ and $E_{z}, D_{z} \sim I_{0}(\rho)$, where $I_{0}$ and $I_{1}$ are Bessel functions. We confine ourselves to Eqs. (4.3) and (4.4) because they are the large-separation limit of the solutions with sources given in Sec. V] and

$$
E_{z}=\frac{1}{4 \pi}\left(1-\rho^{2} / a^{2}\right)
$$

subject to the boundary conditions that $C$ and $E_{z}$ are continuous at $\rho=a$ with the solutions outside, which are $C=-1 / 2 \pi \rho$ and $E_{z}=0$. The electric displacement is

$$
\begin{aligned}
& D_{\rho}=0, \\
& D_{z}=-\frac{1}{\rho} \frac{\partial}{\partial \rho} \rho C(\rho)=\frac{1}{\pi a^{2}} ;
\end{aligned}
$$

it is not continuous at $\rho=a$, since the outside value of $D$ is of course zero.

The action density is (using the field equations)

$$
L=-\frac{1}{2} D^{2}-\frac{1}{2} \mathbf{D} \cdot \mathbf{E}+\epsilon_{\mathrm{vac}}
$$

and the energy density $\mathscr{H}$ is minus this. Explicitly, we find from Eqs. (4.4) and (4.5) that the total energy (per unit length) in the cylinder is

$$
E(a)=\int_{0}^{a} 2 \pi \rho d \rho \mathscr{P}(\rho)=\frac{1}{2 \pi a^{2}}+\frac{1}{16 \pi}-\pi a^{2} \epsilon_{\mathrm{vac}} ;
$$

minimizing the energy with respect to the cylinder radius $a$ leads to the boundary condition determining $a$; namely,

$$
\frac{1}{2 \pi^{2} a^{4}}=-\epsilon_{\mathrm{vac}}
$$


This equation is immediately recognizable as

$$
\left.\left(\frac{1}{2} D^{2}+\frac{1}{2} \mathbf{D} \cdot \mathbf{E}\right)\right|_{\rho=a}=-\epsilon_{\mathrm{vac}} ;
$$

thus it describes the balance between the vacuum pressure outside the cylinder and the electrostatic energy density inside the cylinder. Evidently this is in general the appropriate boundary condition. The total color-electric flux is

$$
\phi=\int \mathbf{D} \cdot d \mathbf{S}=\int_{0}^{a} 2 \pi \rho d \rho \frac{1}{\pi a^{2}}=1 .
$$

The exact, non-Abelian, field equations require the quantization of color-electric flux in the cylinder; the approximate Abelian equations of course do not; to lowest order in $g$ solutions could, in principle, exist for any amount of flux. However the boundary condition that, at the surface, $C$ must be continuous with the outside value of $C=-1 / 2 \pi \rho$ guarantees that the flux comes out to have the right value.

This simple example illustrates how the approximate equations imitate the exact solutions given in I. Comparing the approximate solutions for $C_{\phi}, D_{z}$, and $E_{z}$ with the exact solutions displayed in I shows that the approximation is reasonably good qualitatively, though quantitatively the numerical values can miss by as much as a factor of 2. This gives us a measure of the reliability of the simplified baglike approximation we are using. The major discrepancy between the approximate solution and the exact solution of the nonlinear equations, however, lies in the existence of $E_{\rho}$ and in its dependence on $z$. The approximate $E_{\rho}$ vanishes at $z=0$. In the exact problem, the region over which $E_{\rho}$ vanishes widens, so that in the presence of sources the exact $E_{\rho}$ exists only near the sources and is zero in much of the region between. The approximate $E_{\rho}$, in contrast [as we see here and also in Eq. (5.11)], simply varies linearly between the sources. Nevertheless, the approximate solution gives a rather good imitation of the exact energy density, since $E_{\rho}$ does not contribute to it. We also learn that the appropriate boundary conditions are that the components of $\mathbf{C}$ and $\mathbf{E}$ tangent to the surface are continuous since $E$ vanishes outside, we must have $E_{t}=0$ on the surface. We infer that in general the required boundary conditions at the surface are $D_{n}=0$ and $E_{t}=0$, the first following from Gauss's law $\boldsymbol{\nabla} \cdot \mathbf{D}=0$ and the second from the continuity of $E$.

The magnetic equations inside the cylindrical sourceless cylinder, to lowest order in $g$, are

$$
\begin{aligned}
& \left(\frac{\partial}{\partial \rho} \frac{1}{\rho} \frac{\partial}{\partial \rho} \rho+\frac{\partial^{2}}{\partial z^{2}}\right) B_{\rho}=\frac{\partial C_{0}}{\partial \rho}, \\
& \left(\frac{1}{\rho} \frac{\partial}{\partial \rho} \rho \frac{\partial}{\partial \rho}+\frac{\partial^{2}}{\partial z^{2}}\right) B_{z}=\frac{\partial C_{0}}{\partial z},
\end{aligned}
$$

and

$$
\left(\frac{1}{\rho} \frac{\partial}{\partial \rho} \rho \frac{\partial}{\partial \rho}+\frac{\partial^{2}}{\partial z^{2}}\right) C_{0}-\frac{1}{\rho} \frac{\partial}{\partial \rho} \rho B_{\rho}-\frac{\partial}{\partial z} B_{z}=0,
$$

where we have taken $\mathbf{B}=B_{\rho} \widehat{\mathbf{e}}_{\rho}+B_{z} \widehat{\mathbf{e}}_{z}$, which is the only form consistent with the assumed azimuthal symmetry and the approximate Abelian equations. Outside we have, we recall, $C_{0}=0$ and $\mathbf{B}=b\left(\widehat{\mathbf{e}}_{\rho} T_{1}+\widehat{\mathbf{e}}_{\phi} T_{2}\right)$; these values (together with $\mathbf{C}=-(1 / 2 \pi \rho) \hat{\mathbf{e}}_{\rho} T_{3}$ and $\left.\mathbf{E}=0\right)$ satisfy the exact non-Abelian field equations [for $\mathrm{SU}(2)$ of color]. Thus inside there is no tangential $B$ field, and therefore in particular it cannot be continuous with the outside tangential $B$ across the boundary. The normal component, however, can be continuous, as can $C_{0}$, and the appropriate solutions to (4.8) and (4.9) are

$$
\begin{aligned}
& C_{0}=0, \\
& B_{\rho}=\rho b / a,
\end{aligned}
$$

and

$$
B_{z}=-2 b z / a .
$$

Then

$$
\mathbf{H}=-\frac{\partial C_{0}}{\partial \rho} \widehat{\mathbf{e}}_{\rho}-\frac{\partial C_{0}}{\partial z} \widehat{\mathbf{e}}_{z}=0 ;
$$

therefore the magnetic energy and action density $\frac{1}{2}(\mathbf{B}+\mathbf{H}) \cdot \mathbf{H}$ inside both vanish. As in the electric case, we infer from this simple example that the boundary conditions $H_{t}=0$ and $B_{n}=b$ hold in general at the surface.

\section{THE ABELIAN DUAL SUPERCONDUCTOR WITH SOURCES: LARGE SEPARATION}

Let us next consider purely electric sources, so that $\mathbf{j}=0$. The magnetic field equations then can be satisfied by choosing $\mathbf{B}=\mathbf{H}=C_{0}=0$ and the electric equations are

$$
\frac{1}{f^{2}} \nabla \times \mathbf{D}+\nabla \times \mathbf{E}=0
$$

and

$$
\mathbf{D}=-\frac{\nabla^{2}}{M^{2}} \mathbf{E}
$$

together with (3.17) and (3.19). The action is

$$
\begin{aligned}
S=\int_{V} d^{3} \mathbf{x} N \operatorname{Tr} & -\mathbf{D} \cdot \mathbf{E}-\frac{1}{2 f^{2}} \mathbf{D}^{2} \\
& \left.-\frac{1}{2} \mathbf{E} \frac{\nabla^{2}}{M^{2}} \mathbf{E}\right)+\epsilon_{\mathrm{vac}} V .
\end{aligned}
$$

As the charge density let us take

$$
\rho=\left[q_{1} \delta^{3}\left(\mathbf{x}-\mathbf{x}_{1}\right)+q_{2} \delta^{3}\left(\mathbf{x}-\mathbf{x}_{2}\right)\right] .
$$

For two quarks in an SU(3)-color-singlet state,

$$
q_{1}=-q_{2}=\sqrt{4 / 3} e
$$

where $e$ is the usual Yang-Mills electric coupling constant, so that

$$
e=2 \pi / g \text {. }
$$

Except in the Abelian limit ( $g$ small) we cannot handle sources with noncommuting color matrices at the classical level. It is necessary to quantize the dual gluon field to do this. A discussion of quantization will be deferred to a later publication; here we will limit ourselves to the Abeli- 
an version (5.1)-(5.3), so that $q_{1}$ and $q_{2}$ are simply numbers. We have

$$
\boldsymbol{\nabla} \cdot \mathbf{D}_{s}=\left[q_{1} \delta^{3}\left(\mathbf{x}-\mathbf{x}_{1}\right)+q_{2} \delta^{3}\left(\mathbf{x}-\mathbf{x}_{2}\right)\right]
$$

We choose the quarks to lie on the $z$ axis at a separation $R$ :

$$
\begin{aligned}
\mathbf{D}= & \widehat{\mathbf{e}}_{\boldsymbol{\rho}}\left[-\frac{1}{2 \pi^{2}} \int_{0}^{\infty} k d k\left[q_{1} \cos k(z-R / 2)+q_{2} \cos k(z+R / 2)\right]\left[-K_{1}(k \rho)+I_{1}(k \rho) \frac{K_{1}(k a)}{I_{1}(k a)}\right)\right] \\
& +\widehat{\mathbf{e}}_{\mathbf{z}}\left[\frac{1}{2 \pi^{2}} \int_{0}^{\infty} k d k\left[q_{1} \sin k(z-R / 2)+q_{2} \sin k(z+R / 2)\right]\left[K_{0}(k \rho)+I_{0}(k \rho) \frac{K_{1}(k a)}{I_{1}(k a)}\right]\right],
\end{aligned}
$$

where $K_{0}, K_{1}, I_{0}$, and $I_{1}$ are Bessel functions. The value of the cylinder radius is determined by the pressure balance equation. When $R$ is very large $(R / a \rightarrow \infty)(5.8)$ becomes

$$
\mathrm{D} \rightarrow \frac{\widehat{\mathbf{e}}_{z}}{2 \pi a^{2}}\left[q_{1} \epsilon(z-R / 2)+q_{2} \epsilon(z+R / 2)\right],
$$

where $\epsilon(z)= \pm 1$ according as $z \leqslant 0$.

The corresponding solution for the electric field $\mathbf{E}$ is

$$
\begin{aligned}
\mathbf{E}= & \widehat{\mathbf{e}}_{z}\left[-\frac{\rho M^{2}}{4 \pi^{2}} \int_{0}^{\infty} d k\left[q_{1} \sin k(z-R / 2)+q_{2} \sin k(z+R / 2)\right]\left[-K_{1}(k \rho)+I_{1}(k \rho) \frac{K_{1}(k a)}{I_{1}(k a)}\right]\right] \\
& +\widehat{\mathbf{e}}_{\rho}\left[\frac{\rho M^{2}}{4 \pi^{2}} \int_{0}^{\infty} d k\left[q_{1} \cos k(z-R / 2)+q_{2} \cos k(z+R / 2)\right]\left[K_{0}(k \rho)+I_{0}(k \rho) \frac{K_{1}(k a)}{I_{1}(k a)}\right]\right] .
\end{aligned}
$$

For large $R$

$$
\mathbf{E} \rightarrow \frac{\widehat{\mathbf{e}}_{z} M^{2}}{8 \pi}\left(1-\rho^{2} / a^{2}\right)\left[q_{1} \epsilon(z-R / 2)+q_{2} \epsilon(z+R / 2)\right]-\frac{\widehat{\mathbf{e}}_{\rho} M^{2}}{4 \pi a^{2}}\left(q_{1}|z-R / 2|+q_{2}|z+R / 2|\right) ;
$$

note that $E_{z}$ vanishes at $\rho=a$ as required by the boundary conditions.

The energy contained in the cylinder is readily calculated. When $R$ is large,

$$
V(R)=\frac{1}{2} \int_{V} d^{3} \mathbf{x}\left(\frac{1}{f^{2}} \mathbf{D}^{2}+\mathbf{D} \cdot \mathbf{E}\right)-\epsilon_{\mathrm{vac}} V \rightarrow-R q_{1} q_{2}\left(\frac{1}{2 \pi a^{2} f^{2}}+\frac{M^{2}}{16 \pi}\right)+\infty\left(q_{1}+q_{2}\right)^{2}-\pi a^{2} R \epsilon_{\mathrm{vac}} .
$$

The divergent term comes from the contribution of the cylinder's end caps, and exists only provided the two quarks are not in a color-singlet state. It represents the net flux flowing out to infinity in this case. When in a singlet state, however, $q_{1}+q_{2}=0$, all the flux coming out of one quark is absorbed by the other, and the divergent part vanishes. Only color singlets have finite energy. (In higher orders in $g$, overall color-singlet states can be constructed from nonsinglet quark configurations together with explicit quantum gluons.)

It is also of interest to calculate the dual potential $C$ for this cylindrical case. We find

$$
\mathbf{C}=\widehat{\mathbf{e}}_{\phi}\left[-\frac{1}{2 \pi^{2}} \int_{0}^{\infty} d k\left[q_{1} \sin k(z-R / 2)+q_{2} \sin k(z+R / 2)\right]\left[-K_{1}(k \rho)+I_{1}(k \rho) \frac{K_{1}(k a)-1 / k a}{I_{1}(k a)}\right]\right]
$$

Note that at $\rho=a$,

$$
C=\frac{1}{4 \pi a}\left[q_{1} \epsilon(z-R / 2)+q_{2} \epsilon(z+R / 2)\right],
$$

continuous with the vacuum value outside the cylinder. $\mathbf{D}$ is calculated from $\mathbf{C}$ via $\mathbf{D}=-\boldsymbol{\nabla} \times \mathbf{C}+\mathbf{D}_{s}$. The $-\nabla \times \mathbf{C}$ terms contains a string joining the two quarks, coming from the $1 / k \rho$ singularity in (5.13), and the "string field" $\mathrm{D}_{s}$ is

$$
\mathbf{D}_{s}=\frac{\delta(\rho)}{4 \pi \rho}\left[q_{1} \epsilon(z-R / 2)+q_{2} \epsilon(z+R / 2)\right] \widehat{\mathbf{e}}_{z}
$$

(recall $q_{1}+q_{2}=0$ in the color-singlet state).
For finite separation of the quark sources, the shape is of course not a cylinder, but is rather determined dynamically. The shape, and the interaction energy, can be obtained using a numerical variational calculation, much like what is done in the conventional bag model. ${ }^{5}$ How this is done is described in Sec. VI.

\section{THE ABELIAN DUAL SUPERCONDUCTOR WITH SOURCES: ARBITRARY SEPARATION}

We next want to solve Eqs. (5.1) and (5.2), subject to the boundary conditions that $D_{n}$ and $E_{t}$ vanish at the surface of the region of normal vacuum, for arbitrary separation 
between the sources. The surface position is determined by the pressure balance condition

$$
\frac{1}{2} \mathbf{D} \cdot(\mathbf{D}+\mathbf{E})=-\epsilon_{\mathrm{vac}} \text {. }
$$

Apart from the presence of the field $\mathbf{E}$, and of Eq. (5.2) relating $\mathbf{E}$ and $\mathbf{D}$, this is the same as the usual MIT bag problem. ${ }^{4}$ Several numerical solutions to the MIT bag exist in the literature ${ }^{9,10}$ but, for varying reasons, none of these is exactly suited to the present problem, so we will use a technique which we describe below.

It is convenient to introduce two functions $\phi$ and $\psi$ defined by $\mathbf{D}=\boldsymbol{\nabla} \phi$ and $\mathbf{E}=\boldsymbol{\nabla} \psi$. [This will not yield the most general solution to $\mathrm{Eq}$. (5.1), but it will give the physically relevant one.] In order to minimize the number of parameters for which the numerical problem has to be solved, it is also convenient to introduce scaled variables. We define

$$
\begin{aligned}
& \mathbf{x}=\left(q^{2} /-2 f^{2} \epsilon_{\mathrm{vac}}\right)^{1 / 4} \overline{\mathbf{x}}, \\
& \phi=\left(-2 q^{2} f^{2} \epsilon_{\mathrm{vac}}\right)^{1 / 4} \bar{\phi},
\end{aligned}
$$

and

$$
\psi=q M^{2}\left(\frac{q^{2}}{-2 f^{2} \epsilon_{\mathrm{vac}}}\right)^{1 / 4} \bar{\psi} .
$$

In terms of the scaled distance and fields, the field equations become

$$
\begin{aligned}
& \bar{\nabla}^{2} \bar{\phi}=\delta^{3}\left(\overline{\mathbf{x}}-\overline{\mathbf{x}}_{2}\right), \\
& \bar{\nabla}^{2} \bar{\psi}=-\bar{\phi},
\end{aligned}
$$

where $\overline{\mathbf{x}}_{1}=(0,0, \bar{R} / 2), \overline{\mathbf{x}}_{2}=(0,0,-\bar{R} / 2)$, and the boundary conditions are

$$
\begin{aligned}
& \partial \bar{\phi} / \partial \bar{n}=\bar{\psi}=0, \\
& (\bar{\nabla} \bar{\phi})^{2}+\bar{\nabla} \bar{\phi} \cdot \bar{\nabla} \bar{\psi}=1,
\end{aligned}
$$

on the surface.

The (unscaled) energy is

$$
\begin{aligned}
V(R)= & \frac{q^{3 / 2}}{\left(2 f^{2}\right)^{3 / 4}}\left(-\epsilon_{\mathrm{vac}}\right)^{1 / 4}\left[\bar{V}_{1}(\bar{R})+\bar{V}_{3}(\bar{R})\right] \\
& +\frac{q^{5 / 2} M^{2}}{16 \pi\left(2 f^{2}\right)^{1 / 4}}\left(\frac{1}{-\epsilon_{\mathrm{vac}}}\right)^{1 / 4}\left[\bar{V}_{2}(\bar{R})\right]+V_{0},
\end{aligned}
$$

where we define

$$
\begin{aligned}
& \bar{V}_{1}(\bar{R})=\int_{\bar{V}} d^{3} \bar{x}(\bar{\nabla} \bar{\phi})^{2}, \\
& \bar{V}_{2}(\bar{R})=8 \pi \int_{\bar{V}} d^{3} \bar{x} \bar{\nabla} \bar{\phi} \cdot \bar{\nabla} \bar{\psi}, \\
& \bar{V}_{3}(\bar{R})=\int_{\bar{V}} d^{3} \bar{x}=\bar{V},
\end{aligned}
$$

and $\bar{V}$ is the scaled volume of the region of normal vacuum. Comparing with the analytic solution for large separation given in Sec. V we see that $\bar{V}_{1}, \bar{V}_{2}$, and $\bar{V}_{3}$ all approach $\bar{R}$ as $\bar{R} \rightarrow \infty$. The undetermined constant $V_{0}$ appears in Eq. (6.9) because in calculating $\bar{V}_{1}$ a selfenergy appears only the singular part of which can be uniquely discarded.

These field equations and boundary conditions require the existence of two cusps in the surface. We can calcu- late the fields and the surface shape analytically in the vicinity of the cusps. Let the equations of the surface be $\bar{\rho}=\bar{\rho}(\bar{z})$, where $\bar{\rho}$ and $\bar{z}$ are cylindrical coordinates relative to the axis through the sources, and let the cusp be located on the axis at $\bar{z}=\bar{z}_{0}$. Since $\partial \bar{\phi} / \partial n=0$ on the surface, we have

$$
\frac{d \bar{\rho}(\bar{z})}{d \bar{z}}=\frac{\partial \bar{\phi} / \partial \bar{\rho}}{\partial \bar{\phi} / \partial \bar{z}}
$$

and the boundary condition

$$
(\partial \bar{\phi} / \partial \bar{\rho})^{2}+(\partial \bar{\phi} / \partial \bar{z})^{2}=1
$$

is valid there as well.

Near the cusp, $\partial \bar{\phi} / \partial \bar{z}=1-\alpha\left(\bar{z}-z_{0}\right)$. Hence

$$
(\partial \bar{\phi} / \partial \bar{\rho})^{2}=(d \bar{\rho} / d \bar{z})^{2}=2 \alpha\left(\bar{z}-z_{0}\right) .
$$

Finally, if $\sigma(\bar{z})$ is the line charge density along the axis, which exists for $\bar{z}>z_{0}$, flux conservation requires $2 \pi \bar{\rho} \partial \bar{\rho} / \partial \bar{z}=\sigma$. Putting all this together we see that $\sigma \sim\left(\bar{z}-z_{0}\right)^{2}$ and $\bar{\rho} \sim\left(\bar{z}-z_{0}\right)^{3 / 2}$ near the cusp. [In two dimensions, $\sigma \sim\left(\bar{z}-z_{0}\right)^{1 / 2}$ and $\bar{\rho} \sim\left(\bar{z}-z_{0}\right)^{3 / 2}$.] The fields themselves are then calculated from

$$
\bar{\phi}=-\frac{1}{4 \pi} \int_{z_{0}} \frac{\sigma\left(\bar{z}^{\prime}\right) d \bar{z}^{\prime}}{\left[\left(\bar{z}-\bar{z}^{\prime}\right)^{2}+\bar{\rho}^{2}\right]^{1 / 2}}
$$

and

$$
\bar{\psi}=\frac{1}{8 \pi} \int_{z_{0}} \sigma\left(\bar{z}^{\prime}\right)\left[\left(\bar{z}-\bar{z}^{\prime}\right)^{2}+\bar{\rho}^{2}\right]^{1 / 2} d \bar{z}^{\prime} .
$$

The numerical procedure we shall use now proceeds as follows. We choose trial fields $\bar{\phi}$ and $\bar{\psi}$ containing the sources and the cusp potentials and satisfying the field equations. We also choose a trial surface shape containing the cusps. We next minimize the integrals $\oint(\partial \bar{\phi} / \partial n)^{2} d S$ and $\oint \bar{\psi}^{2} d S$ with respect to the parameters in the trial fields. This yields analytic expressions for parameters in $\bar{\phi}$ and $\bar{\psi}$ in terms of the parameters describing the trial surface. Finally we calculate

$$
\chi^{2} \equiv \frac{\oint d S\left[\left[(\bar{\nabla} \bar{\phi})^{2}-1\right]^{2}+\left[\frac{\partial \bar{\phi}}{\partial n}\right]^{2}+\bar{\psi}^{2}\right]}{\oint d S},
$$

and search numerically on the parameters giving the surface shape to minimize $\chi^{2}$.

The explicit parameterization we use for $\bar{\phi}$ is (in spherical coordinates)

$$
\begin{aligned}
\bar{\phi}= & -\frac{1}{4 \pi}\left[\frac{1}{\left|\overline{\mathbf{x}}-\overline{\mathbf{x}}_{1}\right|}-\frac{1}{\left|\overline{\mathbf{x}}-\overline{\mathbf{x}}_{2}\right|}\right]+\bar{\phi}_{\text {cusp }} \\
& +\sum_{l=1}^{N} A_{l} \bar{r}^{2 l-1} P_{2 l-1}(\cos \theta),
\end{aligned}
$$

where

$$
\begin{aligned}
\bar{\phi}_{\text {cusp }}=\lambda & \int_{z_{0}}^{z_{c}} \frac{\left(\bar{z}^{\prime}-z_{0}\right)^{2}}{\left[\left(\bar{z}^{\prime}-\bar{z}\right)^{2}+\bar{\rho}^{2}\right]^{1 / 2}} d \bar{z}^{\prime} \\
& \left.-\int_{-z_{c}}^{-z_{0}} \frac{\left(\bar{z}^{\prime}+z_{0}\right)^{2}}{\left[\left(\bar{z}^{\prime}-\bar{z}\right)^{2}+\bar{\rho}^{2}\right]^{1 / 2}} d \bar{z}^{\prime}\right] .
\end{aligned}
$$


The parameters here are the $A_{l}$, as well as $z_{c}$ and $\lambda$; the cusp position $z_{0}$ is part of the surface parameterization. Minimization of $\oint(\partial \phi / \partial n)^{2} d S$ with respect to the $A_{l}$ gives a set of linear equations for the $A_{l}$ which are solved analytically on the computer.

For $\bar{\psi}$ we write

$$
\begin{aligned}
\bar{\psi}= & \frac{1}{8 \pi}\left(\left|\overline{\mathbf{x}}-\overline{\mathbf{x}}_{1}\right|-\left|\overline{\mathbf{x}}-\overline{\mathbf{x}}_{2}\right|\right)+\bar{\psi}_{\text {cusp }} \\
& -\sum_{l=1}^{N} A_{l} \bar{r}^{2 l+1} P_{2 l-1}(\cos \theta) /(8 l+2) \\
& +\sum_{l=1}^{N} B_{l} \bar{r}^{2 l-1} P_{2 l-1}(\cos \theta)
\end{aligned}
$$

where

$$
\begin{aligned}
\bar{\psi}_{\text {cusp }}=-\frac{\lambda}{2}[ & \int_{z_{0}}^{z_{c}}\left(\bar{z}^{\prime}-z_{0}\right)^{2}\left[\left(\bar{z}^{\prime}-\bar{z}\right)^{2}+\bar{\rho}^{2}\right]^{1 / 2} d \bar{z}^{\prime}, \\
& \left.-\int_{-z_{c}}^{-z_{0}}\left(\bar{z}^{\prime}+z_{0}\right)^{2}\left[\left(\bar{z}^{\prime}-\bar{z}\right)^{2}+\bar{\rho}^{2}\right]^{1 / 2} d \bar{z}^{\prime}\right) .
\end{aligned}
$$

Again, minimizing $\oint \bar{\psi}^{2} d S$ with respect to the $B_{l}$ determines these parameters.

Finally, the surface shape is written

$$
\bar{r}(\theta)=\frac{\sum_{l=0}^{M} C_{l} P_{2 l}(\cos \theta)+D(\sin \theta)^{2 / 3}}{\left(1+E \cos ^{2} \theta\right)^{1 / 2}},
$$

where $D$ and $E$ describe the cusp shape. (See Haxton and Heller, Ref. 10. This paper actually allows the power of $\sin \theta$ to be arbitrary. However, as we have seen, the correct power to describe the cusp is $\frac{2}{3}$.) The cusp position $z_{0}$ is given by $z_{0}=\bar{r}(\theta=0)$.

The asymptotic solution in Sec. $\mathrm{V}$ tells us that as $\bar{R} \rightarrow \infty, A_{1} \rightarrow 1, B_{1} \rightarrow \frac{1}{8}, C_{0} \rightarrow 1 / \sqrt{\pi}, E \rightarrow-1, D \rightarrow 0$, and the remaining $A_{l}$ and $B_{l}$ all vanish.

The final step of the method now is to use Eqs. (6.13), (6.15), and (6.17) to calculate $\chi^{2}$ and then to minimize this numerically with respect to the remaining parameters $\lambda$, $z_{c}, C_{l}, D$, and $E$.

The method can be tested by comparing its results, in two spatial dimensions, with an analytic calculation given by Giles. ${ }^{11}$ To do this we replace $\bar{r}^{l} P_{l}$ by $\bar{\rho}^{l} \cos l \theta$ [where $\left.\bar{\rho}=\left(\bar{x}^{2}+\bar{z}^{2}\right)^{1 / 2}\right], \quad-1 / 4 \pi\left|\overline{\mathbf{x}}-\overline{\mathbf{x}}_{1}\right|$ by $1 / 2 \pi \ln \left|\overline{\mathbf{x}}-\overline{\mathbf{x}}_{1}\right|$, the cusp potential by its two-dimensional form, and of course we drop $\bar{\psi}$. Figure 1 shows the surface shape obtained by our method and the shape obtained analytically by Giles, at a source separation $\bar{R}=2$. Our $\chi^{2}$ for this case is $3 \times 10^{-5}$. The agreement is evidently excellent. At a separation $\bar{R}=0.123$, our $\chi^{2}$ is $1.6 \times 10^{-5}$ and the two curves in the corresponding figure are indistinguishable.

In our three-dimensional calculations, we usually use six Legendre functions in each of $\bar{\phi}$ and $\bar{\psi}$. We have tried up to ten at $\bar{R}=2$. The $\chi^{2}$ we obtain is in the range of a few times $10^{-5}$. The quantities $\bar{V}_{1}$ and $\bar{V}_{3}$ are quite insensitive to the numbers of parameters; $\bar{V}_{2}$ becomes somewhat sensitive for $\bar{R} \geq 2$. For this reason we have, in our actual results, used the asymptotic form of $\bar{V}_{2}$ beyond $\bar{R}=1$.

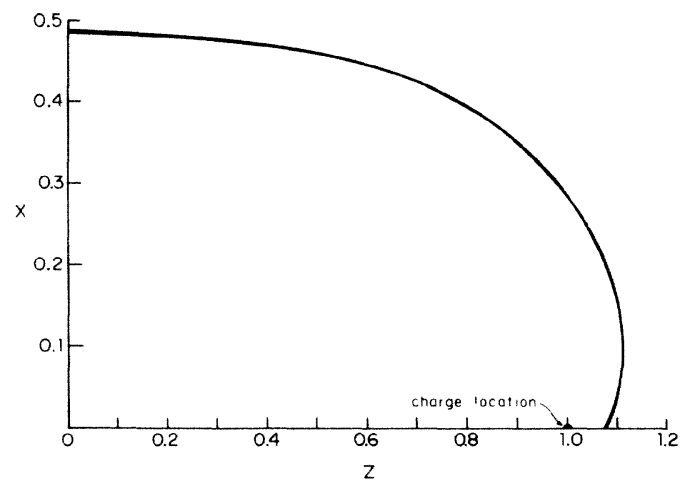

FIG. 1. Comparison of two-dimensional surface shapes (of which only the first quadrant is shown) as calculated by our program with the analytically calculated shape from Ref. 11 . The separation is $\bar{R}=2$. The curves are indistinguishable, except near $z=0$.

For the surface shape five Legendre functions suffice; the shapes are quite insensitive to this number. The five $C_{l}$, plus two cusp parameters in the surface shape, plus the two cusp parameters in $\bar{\phi}$ and $\bar{\psi}$ are the parameters we search on. The surface integrals are evaluated at 20 points, distributed so as to keep the same number of points in the cusp region for each value of $\bar{R}$.

Figures 2 and 3 show the computed surface shapes at $\bar{R}=0.2$ and $\bar{R}=2$. For small $\bar{R}$, the surface is not at all spherical; it is much more like a bagel. (It is very inaccurate to leave out the cusp terms, unless some sort of surface tension is included. A spherical approximation at small $\bar{R}$ does not work for the shape, though it is not too bad for the energy. See Refs. 4 and 9.)

The final step in calculating the static potential is to put the physical dimensions back into the problem, via Eqs. (6.2)-(6.4) and (6.9). From the asymptotic forms $\bar{V}_{1}, \bar{V}_{2}, \bar{V}_{3} \rightarrow \bar{R}$ we see that, as $R \rightarrow \infty$,

$$
V(R) \rightarrow \kappa R,
$$

while from the small $\bar{R}$ limits $\bar{V}_{2}, \bar{V}_{3} \rightarrow 0$ and $\bar{V}_{1} \rightarrow-1 / 2 \pi \bar{R}$ we have, as $R \rightarrow 0$

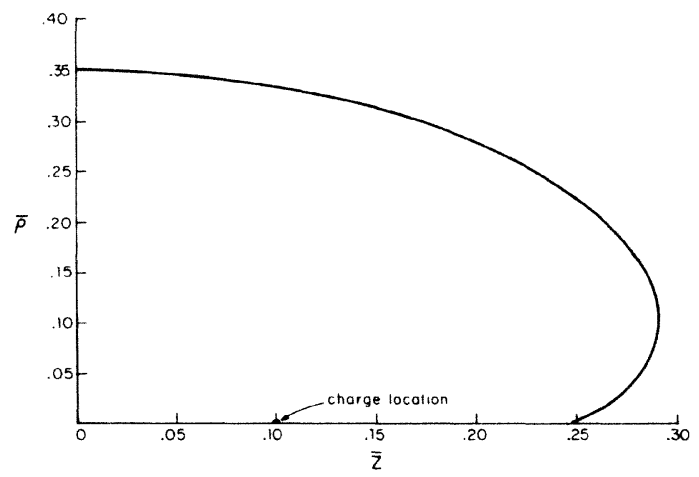

FIG. 2. Surface shape for $\bar{R}=0.2$. Note the different horizontal and vertical scales. 


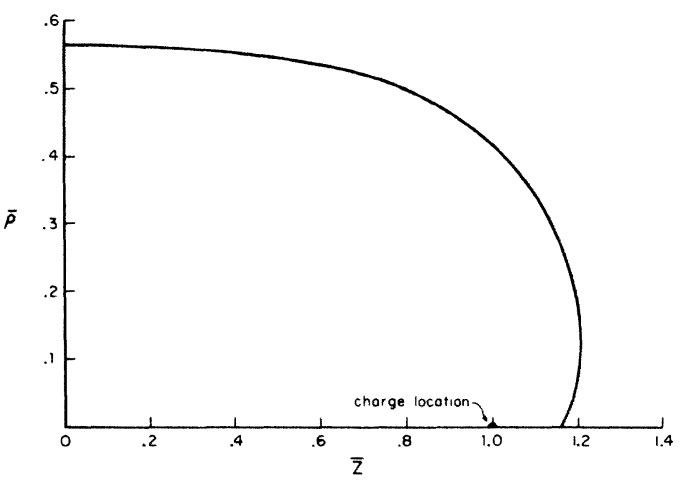

FIG. 3. Surface shape for $\bar{R}=2.0$.

$$
V(R) \rightarrow-\frac{4}{3} \frac{\alpha_{s}}{R},
$$

where the unscaled separation $R$ is

$$
R=\sqrt{\pi} a \bar{R},
$$

the string tension $\kappa$ is

$$
\kappa=\frac{1}{3} \alpha_{s} f^{2} M^{2}+\frac{16}{3} \frac{\alpha_{s}}{a^{2}},
$$

the asymptotic bag radius $a$ is

$$
a=\left(-8 \alpha_{s} / 3 \pi \epsilon_{\mathrm{vac}}\right)^{1 / 4},
$$

and the coupling constant $\alpha_{s}$ is

$$
\frac{4}{3} \alpha_{s}=\frac{q^{2}}{4 \pi f^{2}}
$$

If we take $q=\sqrt{4 / 3} e$, appropriate to two $\mathrm{SU}(3)$ quarks in a color-singlet state, then $\alpha_{s}=e^{2} / 4 \pi f^{2}$. Insisting upon the correct QCD short-range result (an insistence which is perhaps extreme in view of the fact that our Lagrangian is only correct at long range) then requires $f^{2}=1$, as we mentioned in the Introduction. We may write the static potential in terms of $\kappa, a, V_{0}$, and $\alpha_{s}$ in the form

$$
\begin{aligned}
V(R)= & \kappa R-\frac{4}{3} \frac{\alpha_{s}}{R}+V_{0}+\pi^{1 / 2} \kappa a\left[\bar{V}_{2}(\bar{R})-\bar{R}\right] \\
& +\frac{8}{3} \pi^{1 / 2} \frac{\alpha_{s}}{a}\left[\bar{V}_{1}(\bar{R})+\bar{V}_{3}(\bar{R})-2 \bar{V}_{2}(\bar{R})+1 / 2 \pi \bar{R}\right] .
\end{aligned}
$$

This potential can be compared with a phenomenologically determined static potential ${ }^{12}$ to fix numerical values for $\kappa, a, V_{0}$ and $\alpha_{s}$. We find $\kappa=0.24 \mathrm{GeV}^{2}, a=0.77 \mathrm{fm}$, $\alpha_{s}=0.27$, and $V_{0}=-1.25 \mathrm{GeV}$. From these values, and Eqs. (6.20) and (6.21), we can extract some of the input parameters in our Lagrangian. Specifically, we obtain $M_{f} \equiv f M=1.26 \mathrm{GeV}$ and $\left(-\epsilon_{\mathrm{vac}}\right)^{1 / 4}=178 \mathrm{MeV}$. If in addition we choose $f=1$ we also have $M=1.26 \mathrm{GeV}$.

The match between our potential (6.23) and the phenomenological one is excellent. As shown in Fig. 4 the

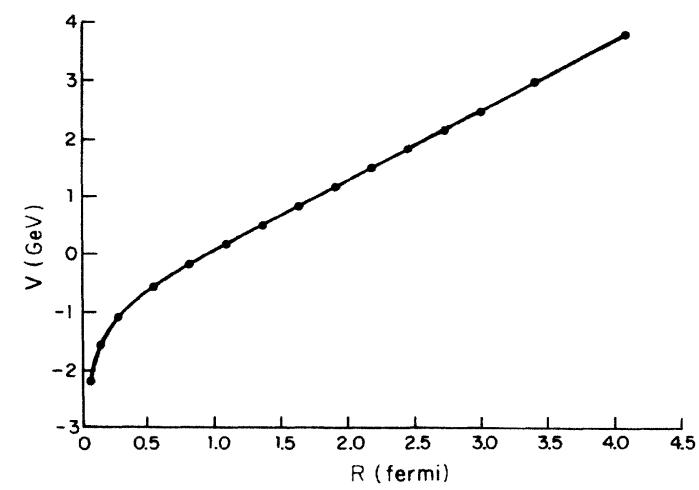

FIG. 4. Comparison of our calculated quark potential versus quark separation with the phenomenological potential of Ref. 12. The two curves are indistinguishable over the entire range.

two curves overlap so closely that they are indistinguishable in the figure over the entire range from 0.07 to $4 \mathrm{fm}$.

\section{CONCLUSION}

We have constructed a method for incorporating heavy-quark sources into the dual-superconductor picture of QCD, and applied this (within a simplifying baglike and Abelian approximation to the actual nonlinear equations of the theory), to the calculation of the static quark potential. The potential we obtain is in remarkably good agreement with phenomenologically determined potentials.

The comparison with phenomenology permits us to obtain the values of some of the constants appearing in the original dual-superconductor Lagrangian; in particular we find that the vacuum energy density is $\epsilon_{\mathrm{vac}}=-(178$ $\mathrm{MeV})^{4}$, and the mass scale $M_{f}$ defined in $I$ is $1.26 \mathrm{GeV}$. If, further, we insist that the dual superconductor Lagrangian interpolates all the way down to very short ranges (a somewhat dubious assumption) then the parameter $f=1$ and so the original mass scale $M$ in the QCD dielectric constant is also $1.26 \mathrm{GeV}$.

These parameters are of course for SU(3) of color, since they are determined from experiment. It is therefore not really possible to compare them with the possible sets of parameters we have obtained before, in the solutions of the exact nonlinear equations of the theory, for cylindri$\mathrm{cal}^{1}$ and spherical ${ }^{5}$ vacuum excitations: they were all for SU(2) of color. In particular, if we used the value of the string tension found here, namely, $0.24 \mathrm{GeV}^{2}$, and the relation $\epsilon_{\mathrm{vac}}=-0.096 \kappa^{2}$ used in Ref. 5, we would get $\epsilon_{\mathrm{vac}}=-(272 \mathrm{MeV})^{4}$, in contrast with the $178 \mathrm{MeV}$ found here. Nevertheless, within this kind of accuracy, the relatively large value of $\boldsymbol{M}_{f}$ we have obtained here suggests, interpolating from Table I of Ref. 5, a glueball mass in the neighborhood of $1.5 \mathrm{GeV}$.

More generally, as the parameters in the effective action (1.2) become better determined, through calculations such as the one described in this paper, the predictive power of the dual-superconductor picture will increase, and we may hope for a relatively complete picture, even quantitatively, of long-range QCD. 
${ }^{1}$ M. Baker, J. S. Ball, and F. Zachariasen, Phys. Rev. D 31, 2575 (1985). We refer to this paper as I. See also V. P. Nair and C. Rosenzweig, Phys. Lett. 135B, 450 (1984).

${ }^{2}$ P. A. M. Dirac, Proc. R. Soc. London A133, 60 (1931).

${ }^{3} \mathrm{~F}$. Englert, in Hadron Structure and Lepton-Hadron Interactions, Cargese 1977, edited by M. Lévy et al. (Plenum, New York, 1979), pp. 503-560.

4See, for example, C. DeTar and J. Donoghue, Annu. Rev. Nucl. Sci. 33, 235 (1983); P. Hasenfratz and J. Kuti, Phys. Rep. 40C, 75 (1978).

${ }^{5}$ M. Baker et al., Phys. Rev. D 33, 1415 (1986).
${ }^{6}$ H. B. Nielsen and P. Olesen, Nucl. Phys. B160, 57 (1979).

${ }^{7}$ V. L. Ginzburg and L. D. Landau, Zh. Eksp. Teor. Fiz. 20, 1064 (1950).

${ }^{8}$ R. Friedberg and T. D. Lee, Phys. Rev. D 18, 2623 (1978),

${ }^{9}$ P. Hasenfratz, R. R. Horgan, J. Kuti, and J. M. Richard, Phys. Lett. 95B, 299 (1980), and Ref. 4.

10W. C. Haxton and L. Heller, Phys. Rev. D 22, 1198 (1980).

${ }^{11}$ R. Giles, Phys. Rev. D 18, 513 (1978).

${ }^{12}$ S. Schmitz, D. Beavis, and P. Kaus, University of California, Riverside Report No. UCR-TH-85-3, 1985 (unpublished). 